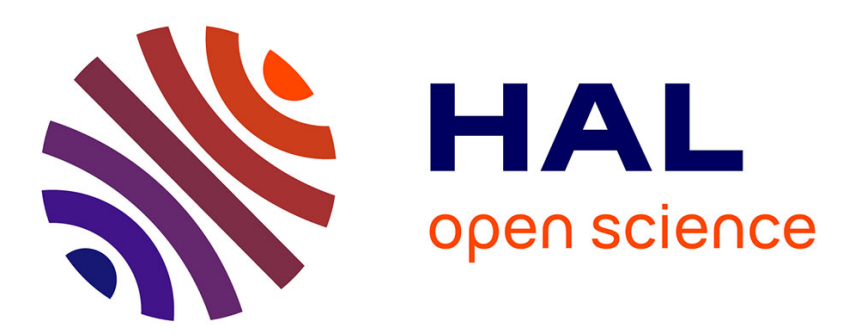

\title{
Let's Listen to the Data: Sonification for Learning Analytics
}

Eric Sanchez, Théophile Sanchez

\section{To cite this version:}

Eric Sanchez, Théophile Sanchez. Let's Listen to the Data: Sonification for Learning Analytics. B. Eagan,; M. Misfeldt; A. Siebert-Evenstone. Advances in Quantitative Ethnography. First International Conference, ICQE 2019, Madison, WI, USA, October 20-22, 2019, Proceedings, 1112, Springer, pp.189-198, 2019, 10.1007/978-3-030-33232-7_16 . hal-03088598

\section{HAL Id: hal-03088598 \\ https://hal.science/hal-03088598}

Submitted on 26 Dec 2020

HAL is a multi-disciplinary open access archive for the deposit and dissemination of scientific research documents, whether they are published or not. The documents may come from teaching and research institutions in France or abroad, or from public or private research centers.
L'archive ouverte pluridisciplinaire HAL, est destinée au dépôt et à la diffusion de documents scientifiques de niveau recherche, publiés ou non, émanant des établissements d'enseignement et de recherche français ou étrangers, des laboratoires publics ou privés. 


\title{
Let's Listen to the Data: Sonification for Learning Analytics
}

\author{
Eric Sanchez ${ }^{1}$ and Théophile Sanchez ${ }^{2}$ \\ 1 University of Fribourg, Fribourg, Switzerland eric.sanchez@unifr.ch \\ 2 Paris-Sud University, Orsay, France theophile.sanchez@u-psud.fr
}

\begin{abstract}
This paper falls in the field of playing analytics. It deals with an empirical work dedicated to explore the potential of data sonification (i.e. the conversion of data into sound that reflects their objective properties or relations). Data sonification is proposed as an alternative to data visualization. We applied data sonification for the analysis of gameplays and players' strategies during a session dedicated to game-based learning. The data of our study (digital traces) was collected from 200 pre-service teachers who played Tamagocours, an online collaborative multiplayer game dedicated to learn the rules (ie. copyright) that comply with the policies for the use of digital resources in an educational context. For one typical individual (parangon) for each of the 5 categories of players, the collected digital traces were converted into an audio format so that the actions that they performed become listenable. A specific software, SOnification of DAta for Learning Analytics (SODA4LA), was developed for this purpose. The first results show that different features of the data can be recognized from data listening. These results also enable for the identification of different parameters that should be taken into account for the sonification of diachronic data. We consider that this study open new perspectives for playing analytics. Thus we advocate for new research aiming at exploring the potential of data sonification for the analysis of complex and diachronic datasets in the field of educational sciences.
\end{abstract}

Keywords: Sonification · Playing Analytics · Data visualisation · GameBased Learning.

\section{Introduction}

Rhythm and music are used as mnemonic means for teaching young students series of items such as days of the week or alphabet. Indeed, representing data through sounds offers the opportunity to benefit from human capacity to memorize or monitor complex temporal audio data. As a result, sonification of data has been recognized as an alternative to data visualization when visualization techniques are insufficient for comprehending certain features in the data or for the analysis of complex datasets. Sonification has already been applied to diverse scientific fields. In this paper we claim that sonification can be applied to 
playing analytics [11 i.e. the record and analysis of players' digital traces during a game session. We present an empirical work dedicated to explore the potential of data sonification for the analysis of players' behaviors. Indeed, one of the main challenges that data analysts face when they want to understand how a game is used consists in identifying the different gameplays performed by players. For this study, 200 pre-service teachers played Tamagocours, a collaborative multiplayer online game dedicated to learn the rules (ie. copyright) that comply with the policies for the use of digital resources in an educational context. We first present a concise overview of the core concepts of sonification. We also describe the game, the data collected, the data processing and the different categories of players identified through a statistical analysis of the data. Third, we describe the results of the sonification of the data and the lessons learned from the work that we carried out with the data collected from the paragons (ie. the typical individuals of the 5 different categories of players that identified with the statistical analysis of the data).

\section{Traditional Approach for Playing Analytics}

Data analysis tools such as KTBS4LA [4] Knime 2, Orange [6, WEKA 9] or R-Studio [15] can be used to conduct analyses on digital data, whether from an educational context or not. These analyses are generally statistical analyses, combined using graphical interfaces or scripts in dedicated languages enabling for data visualization. However, since the temporal aspect of data is essential to analyze the dynamics of knowledge in the context of learning, the time aspect of the data analyzed is not specifically taken into account by these tools. To take this temporal aspect into account, the work carried out by the Learning Analytics community focuses on timestamped traces representing learners' activity with the digital environment and theses traces enable for the visualization of learners' activity played with chronograms (e.g. [13]). Specific patterns might be identified in the traces collected from learners' activity (e.g [13]). These patterns are key indicators of students' strategies. They are important pieces of information for the analysis of the learning process. Tools dedicated to identify these specific patterns have already been developed (e.g. [14]). However, they are not adapted to real time analysis and they do not make difference between patterns that have or have not information value. Thus we think that an alternative to data visualization is needed.

\section{Sonification as an Alternative to Visualization}

Sonification is defined as a systematic and reproducible transformation technique that can be used with different input data to produce sound that reflects objective properties or relations in the data 8 . Thus, sonification refers to audiobased data processing: data relations are transformed into perceived relations in 
an acoustic signal for the purposes of facilitating communication or interpretation [10. Sonification is a recent research field but with already many ancient applications and sonification has been recognized to be a relevant alternative to data visualization. For examples, in a work published in 1961, Speeth demonstrated that auditory presentations of seismic data enable for the discrimination between earthquakes and bomblasts 14 . Different applications have been described. Kramer and al. 10] report successful sonifications such as the Geigercounter, the discovery of a problem with Voyager 2 spacecraft due to high-speed collisions with electromagnetically charged particles. They also report sensory substitutions for visually impaired users of graphical information. More recently, sonification has been used as a mean for the analysis of complex datasets such as trading-data [16] and volcanoes activity [1].

Two main arguments are putted forward for the sonification of data: the limits of users' abilities to interpret visual information and the need to comprehend complex and big data. Indeed, research into auditory perception emphasizes the capacity of the auditory system. Different studies demonstrate that human beings are particularly sensitive to temporal characteristics or changes in sounds over time [7]. Complex and dynamic auditory patterns are generally well perceived and human auditory system organizes sound into perceptually meaningful elements [3]. Human hearing is also well designed to discriminate between periodic and aperiodic events and it enables for the detection of small changes in the frequency of continuous signals. Kramer's work also emphasizes that human hearing enables for discerning relationships or trends in data streams (Kramer, 1994). Thus, sonification appears to be a powerful approach to study complex datasets.

For this preliminary study, we collected data from an empirical work dedicated to explore players's strategies during an online game-based learning session for pre-service teachers. We found that this data has the different characteristics listed above, such as diachronic and periodics events. The complexity and the volume of the information to proceed and analyze also lead us to consider that the data collected was good candidates for sonification.

\section{A Game-Based Learning Case Study}

\subsection{Tamagocours, a Multiplayer Online Game}

Tamagocours is a multiplayer online game dedicated to teach pre-service teachers the legal rules that comply with the policies for the use of digital resources within an educational context [12. Tamagocours is a tamagochi-like game. It is based on a metaphor; a character (called Tamagocours) which needs to be fed with digital educational resources. A team is composed of 2 to 4 players randomly chosen by the system. Each player has to choose a can (which represents a given educational resource) on a shelf, according to its characteristics (eg. date 
of publication) and the format under which this resource will be used (eg. collective projection or online). The selected resources are stored in a fridge before the player feeds the character (the Tamagocours) with this resource. The players have access to the legislation about the copyright for the use of educational resources at any time. The players have also the possibility to chat with their teammates and to discuss about the compliance of one or other resource before feeding the Tamagocours. If a given resource complies with the copyright policies, it enables the character to stay healthy (the Tamagocours becomes green) and the player earns points. Otherwise, the Tamagocours gets sick (red colour) and dies if fed with too many inappropriate resources. The team can replay each level they have lost indefinitely until they reach the following level. The players have also access to the description of the rules of the game and the challenge to be addressed. A session encompasses 5 levels and lasts approximately one hour.

\subsection{An Empirical Study}

We carried out a study aiming at drawing a behavioral and epistemic models of the students (ie. describing the strategies of the players/learners and evaluating to what extent they learn) 12. The research methodology is Design-Based [5] [13. It combines design and analysis within an iterative process carried out in ecological settings. This work implied various stakeholders: researchers, software developers, designers, students, tutors, pedagogical engineers, administrative representatives and a legal expert.

Our methodology is also based on recording and analyzing the digital traces of interaction produced by 200 pre-service teachers who played during a 60 minutes online session. For the analysis of the digital traces we used a statistical procedure based on an orthogonal transformation to convert a set of correlated variables into a set of linearly uncorrelated variables called principal components. This principal component analysis (PCA) enable for drawing 3 axis with significant eigenvalues. PCA was followed by a clustering method (hierarchical cluster analysis) to identify different classes of players depending on their strategies. As a result, the data collected enable to draw behavioral and epistemic models of the players/learners and to distinguish different gameplays. We also developed SOnification of DAta for Learning Analytics (SODA4LA), a software dedicated to the sonification of the data collected for individual typical of the different classes.

\subsection{Five Categories of Players/Gameplays}

One objective of our study consists in describing the different gameplays performed by the players. This has been done through the recording and the analysis 
of the actions performed by the students and the comparison of the typical individuals from each categories of players. We followed different steps for data processing and data analysis:

1. Digital traces are automatically collected when the students play. The different actions performed are recorded (id of the player, click on a specific feature of the interface, chat with a teammate, success or failure...). Time stamped raw data is stored in a csv format file.

2. Raw data is coded according to a theoretical model of play 12. In particular, the messages written by the students are tagged and allocated to different categories depending on their meaning in terms of how the students deal with the knowledge that they are expected to learn.

3. Aggregate data is produced from raw data. The data analysis process combines a principal component analysis followed by a clustering method. This method enables for identifying 5 categories of players according to the actions that they performed during the game session. Thus, these categories describe the different strategies followed by the players.

4. Sonification of coded data is automatically performed with SODA4LA, a software that we developed. A note is automatically allocated to each category of action performed by the player and time intervals between two actions are reduced. One audio file is produced for each player.

5. Data analysis is performed with MuseScore(c), a software enabling to listen to the files and to create a musical score. Data analysis is under the control of a theoretical model of play [12. Hypothesis about students behavior are confirmed or disproved. In the following we will focus on the information conveyed by the audio files for different individuals that are recognized to be archetypal of 5 categories of players. These individuals are named parangons.

6. The conclusions drawn from data analysis are used by researchers to revise their theoretical model. Data is also used by computer scientists for the reengineering of the game. In the future, we plan to offer trainers and learners the opportunity to benefit from the analysis of this data as a mean for trainers to assess students or the opportunity to foster students' awareness (i.e. to reflect about the strategies that they perform during the game session).

In the following, we will examine the sonified data for the 5 categories of players:

- Students who belong to category 1 (p_1, 12,3\%) are named talkative. They send a lot of messages to theirs teammates during a play session. They are also active and perform a lot of actions. 
- Category 2 (p_2, 20,2\%) consists in students named prudent. They mainly feed the Tamagocours with paying attention to the characteristics of the resources that they use. Indeed, they consult the characteristics of the selected items before feeding the Tamagocours. They also often access to the legal documentation available in the game.

- Students from category 3 (p_3, 36\%) are recognized to be efficient. They manage to have a good ratio of success in comparison to failures and they usually pay attention to the characteristics of the resources that they select before feeding the Tamagocours.

- Students from category 4 (p_4, 26,3\%) are named force feeders. They frequently feed the character without paying attention to the characteristics of the resources that they use and the ratio success/failure is below average. It may seem at times as if the player is just using a trial and error approach

- Category 5 (p_5, 5,2\%) consists in students named experts. They make few mistakes. They also pay attention to the characteristics of the resources and collaborate with their teammates. Indeed, they send a lot of messages to express their opinion about the legal rules that have to be respected.

\section{Listening to the Parangons}

In this section we compare different methods for the data sonification and we discuss to what extend each method enable for the identification of specific patterns that are typical from the different identified categories. Thus, the different examples provided are parangons of the different categories of gameplays.

\subsection{Coding the Actions with Sounds}

Sonification consists of transforming data so that they will become listenable by a data analyst. For this exploratory study we decided to take into account only two dimensions: the type of action performed by a player and intervals between two action (the diachronic feature of the data).

Each type of action is replaced with a note (ie. a sound with a specific frequency) from the $\mathrm{C}$ Dorian mode. The choices made are arbitrary and the following audio score indicates how the different actions performed by the players are matched with sounds. The different actions are the following (Fig. 1):

- "chat" (the player send a message to his teammates): "C in octave 4" 
- "showItemCupboard" (the player consults the characteristics of given resource): "D in octave 4"

- "addToFridge" (the player stores a given resources in the fridge): "E-flat in octave 4"

- "feedTamago" (the player feeds the Tamagocours with a given resource): "F in octave 4"

- "help" (consulting information about the game): "G in octave 4

- "helpLink" (consulting the legal library): "A in octave 4"

- "showItem" (the player check the characteristic of a specific resource added by a teammate into the fridge): "B-flat in octave 4"

- "tuto" (the player consults a tutorial about how to play the game): "C in octave 5"

- "removeFromFridge": "D in octave 5"

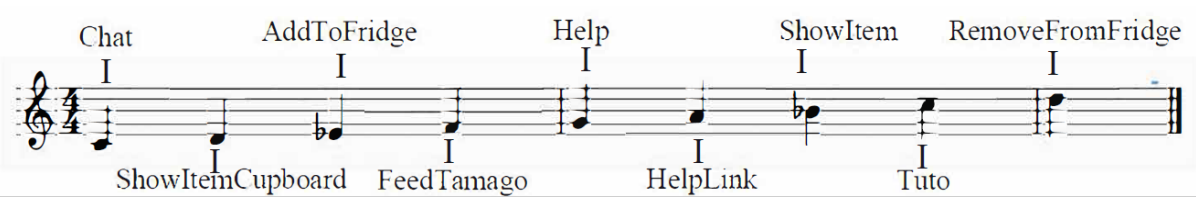

Fig. 1. Key for the coding of the data.

Intervals between two actions have also to be coded. We tried different options: time between 2 notes is equal to the time between 2 actions (real_time), notes are played without taking into account the time between 2 actions (no_time) and time is reduced (compressed) with a log-transformation and rounded to eight possible pitches that are then associated to eight relative note values (duration) $(1 / 4,1 / 3,1 / 2,1,2,3,4$ and 5$)$. The sonification of the data produces melodies with the same tune but with different tempos that illustrate the same gameplay. When the tempo is too low (real_time option), the musical dimension of the data tends to disappears and it becomes difficult to identify a 'music tune'. As a result, it seems to be important that the coding of the data enables for producing sounds that can be considered to be 'music'. We finally selected the third option for the sonification of data in order that the 'music' gives an idea of the tempo of the gameplay without too much silence between two notes. 
This preliminary work shows that the main difficulty faced for the interpretation of the music in terms of gameplay (what is the player's strategy?) consists in giving meaning to the 'music' that is produced. For future work, an option might be to use sounds with more semantic content than notes. For example it is possible to replace a note with the sound made by the character when feed with an appropriate resource. Another option would be to offer data analysts the opportunity to choose how data are sonified. In this regard, an interface dedicated to select relevant parameters would be useful.

\subsection{Identifying Similar Gameplays}

The two examples below are audio files produced with the sonification of the data for 2 different parangons from the same category.

- p1_79171_compressed.wav

- p1_56107_compressed.wav

- p4_62127_compressed.wav

'Music' produced by players from the same category (p_1 talkative) has very similar features. By contrary they differ from the 'music' produced by a 'force feeder' (p4_62127). This observation tends to demonstrate that sonification enables to identify differences among different gameplays at a global level for analysis. It is also plausible that, with some experience and training, a data analyst becomes able to identify a specific gameplay with sonified data.

\subsection{Identifying Specific Patterns}

The five examples below are audio files produced with the sonification of the data produced by 5 different parangons.

- p1_79171_compressed.wav

- p2_61122_compressed.wav

- p3_63131_compressed.wav

- p4_62127_compressed.wav

- p5_4768_compressed.wav 
Listening to the files produced by 5 different parangons confirms that the different gameplays are made 'visible' with music which varies among players. In addition, it is possible to identify some patterns that seem to be typical for the different parangons. For example, a note (D) is played many times for the individual tagged p_1 (fig. 2). This note corresponds to messages sent by the player.

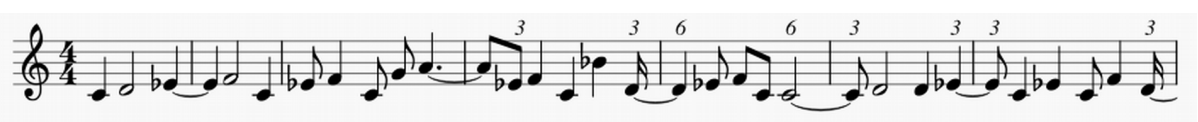

Fig. 2. Sample of the musical score for p1_56107

Fig. 3 shows a similar feature for p1_92206 (another talkative player).

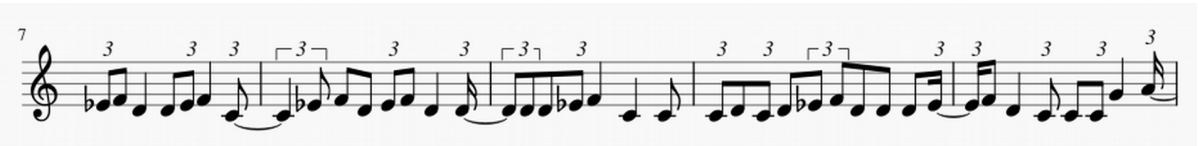

Fig. 3. Sample of the musical score for p1_92206

There is also a clear difference between p2_61122 and p4_62127. For p2_61122, the music is constructed on a pattern of 3 notes whereas a pattern of 2 notes is dominant for player p4_62127. These patterns consist in the principal action that a player needs to perform (consulting the characteristics of a given resource in the shelf, putting this resource into the fridge and feeding the Tamagocours). The first action is missing for p4_62127, force feeders usually do not check the characteristics of a resource that the select before feeding the Tamagocours.

\subsection{Identifying Gameplay Variations}

Though that statistical analysis enables for allocating each player to a specific category, this analysis is based on the average number of variables (actions) for the whole session. Gameplay might change among time and it is important to identify when and how the player's behavior varies. The two following examples show that sonification enable for identifying such changes.

- p2_4984_compressed.wav 
- p3_63131_compressed.wav

For player p2_4984, there is a clear difference of the melody at the end of the file. The melody becomes less monotonous. The data confirm that before 1:20 the majority of actions consist in checking the characteristics of the resources (ShowItemCupboard). After 1:20 he selects more resources (AddToFridge is more frequent) and feed more often the Tamaocours (FeedTamago). This change in the player behavior is well perceived with auditory information. A similar shift from one strategy to another is easily identified for player p3_63131. For this 'prudent' player, the shift occurs around 0:18 and correspond to an enrichment of the actions performed with more ChatAction and HelpLink.

\section{Conclusion}

It becomes apparent from this study that sonification enable for the identification of different features of our data: specific melodies linked with specific gameplay and variation of gameplay among time. Though that this preliminary work tends to demonstrate that sonification might be relevant for playing analytics, numerous challenges remain. First, there is a need for a specific software dedicated to match actions performed by players and sounds according to the need of the data-analysts. This tool should also provide with different options for the coding of the data. For example, the choice of different musical parameters (ie. note pitch, value, tempo, timber) should be under the control of data-analysts. In addition, it is plausible that sonified data are more easy to interpret (or at least to discriminate) if the produced audio file is close to music. In this regard, the musical competences of data-analysts might be important both for the coding of the data and for their interpretation. We need to imagine how to train researchers in charge of making meaning with sonified data. Another challenge lies in the fact that it is important to use sounds that have some semantic content. Within the game, some actions performed by the player such as success or failures for feeding the Tamagocours produce sounds that could be used for the sonification of data.

According to our knowledge, sonification has never been applied in educational research and playing analytics. However, it appears to be a powerful approach for studying complex systems such as learning setting. This empirical works aims to explore this idea. We think that new perspectives are now open. For example, it is extremely difficult to capture complex features such as peer-topeer collaboration with technology enhanced learning systems. We hypothesize that data produced by different learners involved in collaborative learning will produce synchronized and harmonious musical phrases while data collected when learners act independently should produce cacophonic sounds. 
New radical ideas are needed for playing analytics and learning analytics more broadly. We want to drastically reverse the traditional approach that is actually based on data visualization. Thus, we advocate for new researches aiming at exploring the potential of data sonification for the analysis of complex and diachronic datasets in the field of educational sciences. Indeed, we still need to build theories to guide decisions about how to sonify the data.

\section{Supplementary Material}

Scores, audio files, and midi files are available here: https://drive.google.com/open?id=1SLMg071XR7Mn14iIV7PdlkstHXGejRa4

\section{References}

1. Salvatore Avanzo, Roberto Barbera, Francesco De Mattia, Giuseppe La Rocca, Mariapaola Sorrentino, and Domenico Vicinanza. Data sonification of volcano seismograms and sound/timbre reconstruction of ancient musical instruments with grid infrastructures. Procedia Computer Science, 1(1):397-406, 2010.

2. Michael R Berthold, Nicolas Cebron, Fabian Dill, Thomas R Gabriel, Tobias Kötter, Thorsten Meinl, Peter Ohl, Kilian Thiel, and Bernd Wiswedel. Knime-the konstanz information miner: version 2.0 and beyond. AcM SIGKDD explorations Newsletter, 11(1):26-31, 2009.

3. AS Bregman. Auditory scene analysis: The perceptual organization of sound. cambridge, ma, us, 1990.

4. Rémi Casado, Nathalie Guin, Pierre-Antoine Champin, and Marie Lefevre. ktbs4la: une plateforme d'analyse de traces fondée sur une modélisation sémantique des traces. 2017.

5. Design-Based Research Collective. Design-based research: An emerging paradigm for educational inquiry. Educational Researcher, 32(1):5-8, 2003.

6. Janez Demšar, Tomaž Curk, Aleš Erjavec, Črt Gorup, Tomaž Hočevar, Mitar Milutinovič, Martin Možina, Matija Polajnar, Marko Toplak, Anže Starič, et al. Orange: data mining toolbox in python. The Journal of Machine Learning Research, 14(1):2349-2353, 2013.

7. Stephen Handel. Listening: An introduction to the perception of auditory events. The MIT Press, 1993.

8. Thomas Hermann, Andy Hunt, and John G Neuhoff. The sonification handbook. Logos Verlag Berlin, Germany, 2011.

9. Geoffrey Holmes, Andrew Donkin, and Ian H Witten. Weka: A machine learning workbench. 1994.

10. Gregory Kramer, BN Walker, Terri Bonebright, Perry Cook, J Flowers, Nadine Miner, John Neuhoff, R Bargar, S Barrass, J Berger, et al. The sonification report: Status of the field and research agenda. report prepared for the national science foundation by members of the international community for auditory display. International Community for Auditory Display (ICAD), Santa Fe, NM, 1999. 
11. Eric Sanchez and Nadine Mandran. Exploring competition and collaboration behaviors in game-based learning with playing analytics. In European Conference on Technology Enhanced Learning, pages 467-472. Springer, 2017.

12. Eric Sanchez, Valérie Martinez-Emin, and Nadine Mandran. Jeu-game, jeu-play, vers une modélisation du jeu. une étude empirique à partir des traces numériques dinteraction du jeu tamagocours. Sciences et Technologies de l'Information et de la Communication pour l'Éducation et la Formation, 22(1):9-44, 2015.

13. Eric Sanchez, Réjane Monod-Ansaldi, Caroline Vincent, and Sina SafadiKatouzian. A praxeological perspective for the design and implementation of a digital role-play game. Education and Information technologies, 22(6):2805-2824, 2017.

14. Sheridan Dauster Speeth. Seismometer sounds. The Journal of the Acoustical Society of America, 33(7):909-916, 1961.

15. RStudio Team et al. Rstudio: integrated development for r. RStudio, Inc., Boston, MA URL http://www. rstudio. com, 42:14, 2015.

16. David Worrall. Using sound to identify correlations in market data. In Auditory Display, pages 202-218. Springer, 2009. 